\title{
Analytical Bending Solutions of Orthotropic Rectangular Thin Plates with Two Adjacent Edges Free and the Others Clamped or Simply Supported Using Finite Integral Transform Method
}

\author{
Qian Xu, ${ }^{1}$ Zhong Yang, ${ }^{1}$ Salamat Ullah, ${ }^{1}$ Zhang Jinghui ${ }^{(D)},{ }^{1}$ and Yuanyuan Gao ${ }^{2}$ \\ ${ }^{1}$ Faculty of Infrastructure Engineering, Dalian University of Technology, Dalian 116024, China \\ ${ }^{2}$ Key Laboratory of Green Construction and Intelligent Maintenance for Civil Engineering of Hebei Province, Yanshan University, \\ Qinhuangdao 066004, China
}

Correspondence should be addressed to Zhang Jinghui; zhangjinghui653@mail.dlut.edu.cn

Received 6 March 2020; Revised 25 March 2020; Accepted 11 April 2020; Published 11 May 2020

Academic Editor: Yinshan Tang

Copyright $\odot 2020$ Qian Xu et al. This is an open access article distributed under the Creative Commons Attribution License, which permits unrestricted use, distribution, and reproduction in any medium, provided the original work is properly cited.

For the first time, the finite integral transform method is introduced to explore the accurate bending analysis of orthotropic rectangular thin plates with two adjacent edges free and the others clamped or simply supported. Previous solutions mostly focused on plates with simply supported and clamped edges, but the existence of free corner makes the solution procedure much complex to solve by conventional inverse/semi-inverse methods. Compared with the conventional methods, the employed method eliminates the need to preselect the deflection function, which makes it more reasonable and theoretical for calculating the mechanical responses of the plates. Moreover, the approach used can also analyze static problems of moderately thick plates and thick plates with the same boundary conditions investigated in this article. Finally, comprehensive analytical results obtained in this paper illuminate the validity of the proposed approach by comparing with the previous literature and finite element method by using (ABAQUS) software.

\section{Introduction}

Due to the better structural performance such as superior strength-to-weight ratios and stiffness-to-weight ratios, composite materials are widely applied in engineering fields such as mechanical engineering, civil and structural engineering, naval, and aerospace. Orthotropic rectangular plate is considered as the fundamental structural application of composite materials. Thus, the research on the mechanical behavior of orthotropic plate aroused the interest of scientists and engineers for more than a century.

Literature surveys reveal that numerical methods such as finite difference method [1], spline element method [2], boundary element method [3], meshless method [4], finite element method (FEM) [5], boundary particle method [6], isogeometric collocation method [7], discrete singular convolution method [8-11], and differential quadrature method $[12,13]$ are competent to analyze the bending of plates with different edge conditions, loading patterns, and material properties. However, the aforementioned numerical methods satisfy the engineering requirements with acceptable error, but approximate solution is obtained, which is the main disadvantage of the numerical methods.

Compared with numerical methods, analytical solution is relatively sparse, which is due to the mathematical complexity in choosing proper trial function to satisfy the governing equation and the boundary conditions simultaneously. It is known that the classic traditional semi-inverse methods [14-17] such as Navier solution and Levy solution can only deal with the static problem of plate with two opposite simply supported edges. Superposition method [18] can be applied for static problems of plates with various boundary conditions. However, the method involves complex solving procedure.

Among the combinations of boundary conditions of rectangular plate, it is very difficult to obtain analytical solution for plate with at least two adjacent edges free. Most previous research studies dealt with the plate with simply or clamped boundary conditions; however, the existence of two adjacent free edges creates a free corner, which eventually 
makes the solution procedure much complex. The classical superposition method cannot deal with the title problem because of requiring the plate deflections to vanish at the free corner. The existing solutions are very few, and the solution procedure is much more difficult which needs a thorough knowledge of mathematics and mechanics. Recently, a Green's function approach is utilized to solve the free vibration problems of circular thin plates [19-21]. This approach allows obtaining the analytical frequency equations as power series fast convergent to exact eigenvalues for different number of nodal diameters. The symplectic superposition method is developed which is the combination of superposition method as stated above and symplectic elasticity approach [22-25], and applied systematically to the bending [26, 27], buckling [28, 29], and free vibration $[30,31]$ problems of plate. This method has attracted wide attention, including plate; it is also applicable to solve shell problems [32]. However, the method involves complex mathematical manipulations, which require skilled personnel in the fields of mathematics and mechanics. Therefore, researchers are still exploring new analytical methods to analyze the title problem with a more effective way and to develop accurate analytical solutions for validating other numerical/approximate solutions.

Recently, the finite integral transform method [33], an effective mathematical method, is developed which is successfully implemented to solve the bending [34-37] and free vibration [38-40] plate problems with different boundary conditions. Unfortunately, there is no report available which presents the solution of the title problem using finite integral transform. For the reason, this study adopts a simpler and more general, finite integral transformation to investigate the title problem. In the solution process, after finite integral transformation, using some inherent properties of the integral kernel, the bending governing equation is transformed into a fully regular infinite system of simultaneous linear algebraic equations with the unknowns determined by satisfying associated boundary conditions. Then, through some mathematical manipulation, the analytical solution is elegantly achieved in a straightforward procedure. "Compared with the traditional semi-inverse methods (e.g., the Navier method, Levy method, and superposition method), the present one is simpler and more rational. A semi-inverse method normally fails to yield a unified solution procedure since it requires case-by-case trial functions to satisfy both the governing equation and boundary conditions. By using finite integral transformation, the high-order partial differential equation is transformed into a system of linear algebraic equations, and the solution of these equations is achieved in a straightforward way. The present results are believed to present a benchmark for validation of other numerical and analytical methods and can be useful for engineers and scientists for academic and practical applications. The succinct but effective technique presented in this study may provide an easy-to-implement theoretical tool to seek more analytic solutions of buckling and free vibration problems of thin plates."

The three complex boundary value problems, i.e., CCFF, CSFF, and SSFF, are studied, where F denotes free edge, $\mathrm{S}$ denotes simply supported edge, and $\mathrm{C}$ denotes clamped edge, and the boundary conditions are taken in clockwise direction.

\section{Application of Finite Integral Transformation for Bending Analysis of Orthotropic Rectangular Thin Plates}

Figure 1 shows the orthotropic thin plate with dimensions of $a \times b \times h$. The bending governing equation of classical Kirchhoff plate theory is expressed as follows [37]:

$$
D_{x} \frac{\partial^{4} W(x, y)}{\partial x^{4}}+2 H \frac{\partial^{4} W(x, y)}{\partial x^{2} \partial y^{2}}+D_{y} \frac{\partial^{4} W(x, y)}{\partial y^{4}}=q(x, y),
$$

where $W(x, y)$ and $q(x, y)$ are the deflection of plate midplane and the distributed transverse load, respectively; $D_{x}$ and $D_{y}$ are the flexural rigidities in the $x$ and $y$ directions, respectively; $H=D_{1}+2 D_{x y}$ is defined as the effective torsional rigidity, where $D_{x y}$ is the torsional rigidity, $D_{1}=$ $\mu_{y} D_{x}=\mu_{x} D_{y}$ is defined with Poisson's ratios $\mu_{x}$ and $\mu_{y}$; the internal forces of orthotropic plates can be expressed in terms of $W(x, y)$ as follows:

$$
\begin{aligned}
M_{x} & =-D_{x}\left(\frac{\partial^{2} W}{\partial x^{2}}+\mu_{y} \frac{\partial^{2} W}{\partial y^{2}}\right), \\
M_{y} & =-D_{y}\left(\frac{\partial^{2} W}{\partial y^{2}}+\mu_{x} \frac{\partial^{2} W}{\partial x^{2}}\right), \\
M_{x y} & =-2 D_{x y} \frac{\partial^{2} W}{\partial x \partial y}, \\
Q_{x} & =-\frac{\partial}{\partial x}\left(D_{x} \frac{\partial^{2} W}{\partial x^{2}}+H \frac{\partial^{2} w}{\partial y^{2}}\right), \\
Q_{y} & =-\frac{\partial}{\partial y}\left(D_{y} \frac{\partial^{2} W}{\partial y^{2}}+H \frac{\partial^{2} W}{\partial x^{2}}\right), \\
V_{x} & =-\frac{\partial}{\partial x}\left[D_{x} \frac{\partial^{2} W}{\partial x^{2}}+\left(H+2 D_{x y}\right) \frac{\partial^{2} W}{\partial y^{2}}\right], \\
V_{y} & =-\frac{\partial}{\partial y}\left[D_{y} \frac{\partial^{2} W}{\partial y^{2}}+\left(H+2 D_{x y}\right) \frac{\partial^{2} W}{\partial x^{2}}\right] .
\end{aligned}
$$

The CCFF rectangular plate is clamped at two adjacent edges $x=0$ and $y=0$, and the other edges are free; the boundary conditions of the plate are as follows:

$$
\begin{gathered}
\left.W\right|_{x=0}=\left.W\right|_{y=0}=0,\left.\quad 2 D_{x y} \frac{\partial W^{2}}{\partial x \partial y}\right|_{x=a, y=b}=0, \\
\left.\frac{\partial W}{\partial x}\right|_{x=0}=0,\left.\quad M_{x}\right|_{x=a}=\left.V_{x}\right|_{x=a}=0, \\
\left.\frac{\partial W}{\partial y}\right|_{y=0}=0,\left.\quad M_{y}\right|_{y=b}=\left.V_{y}\right|_{y=b}=0 .
\end{gathered}
$$




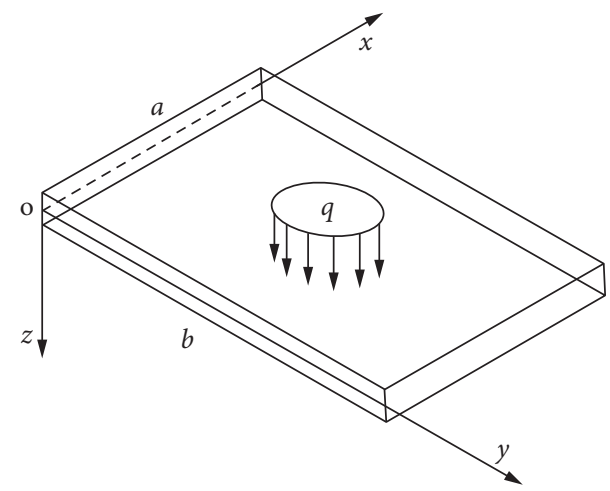

Figure 1: Schematic illustration of thin plate.

$$
\begin{array}{r}
W_{m n}=\int_{0}^{a} \int_{0}^{b} W(x, y) \sin \frac{\alpha_{m} x}{2} \sin \frac{\beta_{n} y}{2} \mathrm{~d} x \mathrm{~d} y \\
(m=1,3,5, \ldots, n=1,3,5, \ldots) .
\end{array}
$$

The inversion is expressed as

$$
W(x, y)=\frac{4}{a b} \sum_{m=1,3}^{\infty} \sum_{n=1,3}^{\infty} W_{m n} \sin \frac{\alpha_{m} x}{2} \sin \frac{\beta_{n} y}{2},
$$

where $\alpha_{m}=m \pi / a$ and $\beta_{n}=n \pi / b$.

The high-order partial derivatives of $W(x, y)$ in equation (1) are derived as follows:

If $W(x, y)$ is a function of the two independent variables $x$ and $y$, defined in a rectangle $0 \leq x \leq a, 0 \leq y \leq b$, the double finite sine integral transform is described as follows:

$$
\begin{aligned}
& \int_{0}^{a} \int_{0}^{b} \frac{\partial^{4} W}{\partial x^{4}} \sin \frac{\alpha_{m} x}{2} \sin \frac{\beta_{n} y}{2} \mathrm{~d} x \mathrm{~d} y \\
& =\left(\frac{\alpha_{m}}{2}\right)^{4} W_{m n}+\int_{0}^{b}\left[\begin{array}{c}
\left.(-1)^{(m-1 / 2)} \frac{\partial^{3} W}{\partial x^{3}}\right|_{x=a}+\left.\frac{\alpha_{m}}{2} \frac{\partial^{2} W}{\partial x^{2}}\right|_{x=0} \\
-\left.(-1)^{(m-1 / 2)}\left(\frac{\alpha_{m}}{2}\right)^{2} \frac{\partial W}{\partial x}\right|_{x=a}-\left.\left(\frac{\alpha_{m}}{2}\right)^{3} W\right|_{x=0}
\end{array}\right] \sin \frac{\beta_{n} y}{2} \mathrm{~d} y . \\
& \int_{0}^{a} \int_{0}^{b} \frac{\partial^{4} W}{\partial y^{4}} \sin \frac{\alpha_{m} x}{2} \sin \frac{\beta_{n} y}{2} \mathrm{~d} x \mathrm{~d} y \\
& =\left(\frac{\beta_{n}}{2}\right)^{4} W_{m n}+\int_{0}^{a}\left[\begin{array}{c}
\left.(-1)^{(n-1 / 2)} \frac{\partial^{3} W}{\partial y^{3}}\right|_{y=b}+\left.\frac{\beta_{n}}{2} \frac{\partial^{2} W}{\partial y^{2}}\right|_{y=0} \\
-\left.(-1)^{(n-1 / 2)}\left(\frac{\beta_{n}}{2}\right)^{2} \frac{\partial W}{\partial y}\right|_{y=b}-\left.\left(\frac{\beta_{n}}{2}\right)^{3} W\right|_{y=0}
\end{array}\right] \sin \frac{\alpha_{m} x}{2} \mathrm{~d} x .
\end{aligned}
$$

The second term of equation (1) can be divided into two parts. In the first part, we consider the partial derivative of $x$ first:

$$
\begin{aligned}
\int_{0}^{a} \int_{0}^{b} \frac{\partial^{4} W}{\partial x^{2} \partial y^{2}} \sin \frac{\alpha_{m} x}{2} \sin \frac{\beta_{n} y}{2} \mathrm{~d} x \mathrm{~d} y \\
=\left(\frac{\alpha_{m}}{2}\right)^{2}\left(\frac{\beta_{n}}{2}\right)^{2} W_{m n}+\int_{0}^{b}\left[\left.(-1)^{(m-1 / 2)} \frac{\partial^{3} W}{\partial x \partial y^{2}}\right|_{x=a}\right. \\
\left.\quad+\left.\frac{\alpha_{m}}{2} \frac{\partial^{2} W}{\partial y^{2}}\right|_{x=0}\right] \sin \frac{\beta_{n} y}{2} \mathrm{~d} y \\
\quad-\left(\frac{\alpha_{m}}{2}\right)^{2} \int_{0}^{a}\left[\left.(-1)^{(n-1 / 2)} \frac{\partial W}{\partial y}\right|_{y=b}+\left.\frac{\beta_{n}}{2} W\right|_{y=0}\right] \sin \frac{\alpha_{m} x}{2} \mathrm{~d} x .
\end{aligned}
$$
of $y$ :

$$
\begin{aligned}
& \int_{0}^{a} \int_{0}^{b} \frac{\partial^{4} W}{\partial x^{2} \partial y^{2}} \sin \frac{\alpha_{m} x}{2} \sin \frac{\beta_{n} y}{2} \mathrm{~d} x \mathrm{~d} y \\
& =\left(\frac{\alpha_{m}}{2}\right)^{2}\left(\frac{\beta_{n}}{2}\right)^{2} W_{m n}+\int_{0}^{a}\left[\left.(-1)^{(n-1 / 2)} \frac{\partial^{3} W}{\partial x^{2} \partial y}\right|_{y=b}\right. \\
& \left.\quad+\left.\frac{\beta_{n}}{2} \frac{\partial^{2} W}{\partial x^{2}}\right|_{y=0}\right] \sin \frac{\alpha_{m} x}{2} \mathrm{~d} x \\
& \quad-\left(\frac{\beta_{n}}{2}\right)^{2} \int_{0}^{b}\left[\left.(-1)^{(m-1 / 2)} \frac{\partial W}{\partial x}\right|_{x=a}+\left.\frac{\alpha_{m}}{2} W\right|_{x=0}\right] \sin \frac{\beta_{n} y}{2} \mathrm{~d} y .
\end{aligned}
$$

By applying one-dimensional finite sine integral transform on the effective shearing forces $V_{x}$ of edge $x=a$, we will obtain 


$$
\begin{aligned}
& \left.D_{x} \int_{0}^{b} \frac{\partial^{3} W}{\partial x^{3}}\right|_{x=a} \sin \frac{\beta_{n} y}{2} \mathrm{~d} y \\
& \quad=-\left.\left(H+2 D_{x y}\right) \int_{0}^{b} \frac{\partial^{3} W}{\partial x \partial y^{2}}\right|_{x=a} \sin \frac{\beta_{n} y}{2} \mathrm{~d} y \\
& \quad=\left.\left(H+2 D_{x y}\right)\left(\frac{\beta_{n}}{2}\right)^{2} \int_{0}^{b} \frac{\partial W}{\partial x}\right|_{x=a} \sin \frac{\beta_{n} y}{2} \mathrm{~d} y .
\end{aligned}
$$

Similarly, after employing one-dimensional finite sine integral transform on the effective shearing forces $V_{y}$ of edge $y=b$, we will obtain

$$
\begin{aligned}
& \left.D_{y} \int_{0}^{a} \frac{\partial^{3} W}{\partial y^{3}}\right|_{y=b} \sin \frac{\alpha_{m} x}{2} \mathrm{~d} x \\
& =-\left.\left(H+2 D_{x y}\right) \int_{0}^{a} \frac{\partial^{3} W}{\partial x^{2} \partial y}\right|_{y=b} \sin \frac{\alpha_{m} x}{2} \mathrm{~d} x \\
& =\left.\left(H+2 D_{x y}\right)\left(\frac{\alpha_{m}}{2}\right)^{2} \int_{0}^{b} \frac{\partial W}{\partial y}\right|_{y=b} \sin \frac{\alpha_{m} x}{2} \mathrm{~d} x .
\end{aligned}
$$

$q_{m n}$ is defined as the transform of the load function $q(x, y)$ :

$$
q_{m n}=\int_{0}^{a} \int_{0}^{b} q(x, y) \sin \frac{\alpha_{m} x}{2} \sin \frac{\beta_{n} y}{2} \mathrm{~d} x \mathrm{~d} y .
$$

By substituting equations (7)-(13) and boundary condition of equation (4a) into equation (1), we can obtain the following equation:

$$
\begin{aligned}
& \left.D_{x} \frac{\alpha_{m}}{2} \int_{0}^{b} \frac{\partial^{2} W}{\partial x^{2}}\right|_{x=0} \sin \frac{\beta_{n} y}{2} \mathrm{~d} y+\left.D_{y} \frac{\beta_{n}}{2} \int_{0}^{a} \frac{\partial^{2} W}{\partial y^{2}}\right|_{y=0} \sin \frac{\alpha_{m} x}{2} \mathrm{~d} x \\
& -\left.(-1)^{\frac{m-1}{2}} D_{x}\left[\left(\frac{\alpha_{m}}{2}\right)^{2}+\mu_{y}\left(\frac{\beta_{n}}{2}\right)^{2}\right] \int_{0}^{b} \frac{\partial W}{\partial x}\right|_{x=a} \sin \frac{\beta_{n} y}{2} \mathrm{~d} y \\
& -\left.(-1)^{\frac{n-1}{2}} D_{y}\left[\mu_{x}\left(\frac{\alpha_{m}}{2}\right)^{2}+\left(\frac{\beta_{n}}{2}\right)^{2}\right] \int_{0}^{a} \frac{\partial W}{\partial y}\right|_{y=b} \sin \frac{\alpha_{m} x}{2} \mathrm{~d} x \\
& +\left[D_{x} \frac{\alpha_{m}^{4}}{16}+H \frac{\alpha_{m}^{2} \beta_{n}^{2}}{8}+D_{y} \frac{\beta_{n}^{4}}{16}\right] W_{m n}=q_{m n} .
\end{aligned}
$$

Some parts of equation (14) are definite integral, which are constants. Let

$$
\left\{\begin{array}{l}
I_{m}=\left.\int_{0}^{a} \frac{\partial W}{\partial y}\right|_{y=b} \sin \frac{\alpha_{m} x}{2} \mathrm{~d} x, J_{m}=\left.\int_{0}^{a} \frac{\partial^{2} W}{\partial y^{2}}\right|_{y=0} \sin \frac{\alpha_{m} x}{2} \mathrm{~d} x \\
K_{n}=\left.\int_{0}^{b} \frac{\partial W}{\partial x}\right|_{x=a} \sin \frac{\beta_{n} y}{2} \mathrm{~d} y, L_{n}=\left.\int_{0}^{b} \frac{\partial^{2} W}{\partial x^{2}}\right|_{x=0} \sin \frac{\beta_{n} y}{2} \mathrm{~d} y
\end{array}\right.
$$

The unidentified constants $J_{m}$ and $L_{n}$ have evident physical meaning. When the plate is clamped, or simply supported at edges $x=0, y=0$, we can easily obtain the following equation:

$$
\left.\frac{\partial^{2} W}{\partial y^{2}}\right|_{x=0}=0,\left.\quad \frac{\partial^{2} W}{\partial x^{2}}\right|_{y=0}=0 .
$$

Substitution of equation (16) into $J_{m}$ and $L_{n}$ leads to

$$
\begin{aligned}
& J_{m}=\left.\int_{0}^{a}\left(\frac{\partial^{2} W}{\partial y^{2}}+\mu_{x} \frac{\partial^{2} W}{\partial x^{2}}\right)\right|_{y=0} \sin \frac{\alpha_{m} x}{2} \mathrm{~d} x, \\
& L_{n}=\left.\int_{0}^{b}\left(\frac{\partial^{2} W}{\partial x^{2}}+\mu_{y} \frac{\partial^{2} W}{\partial y^{2}}\right)\right|_{x=0} \sin \frac{\beta_{n} y}{2} \mathrm{~d} y .
\end{aligned}
$$

Obviously, the integrands of $-D_{y} J_{m}$ and $-D_{x} L_{n}$ are Fourier coefficients of the bending moments of edges $y=0$ and $x=0$, respectively. Similarly, $I_{m}$ and $K_{n}$ are Fourier coefficients of the slopes of free edges $y=b$ and $x=a$, respectively. As to the simply supported edges, the corresponding unknowns will be zero. Accordingly, equation (14) is expressed by an unidentified constants $I_{m}, J_{m}, K_{n}$, and $L_{n}$ as follows:

$$
\begin{aligned}
W_{m n}= & C_{m n}\left[q_{m n}+(-1)^{(n-1 / 2)} D_{y} R_{m n} I_{m}-D_{y} \frac{\beta_{n}}{2} J_{m}\right. \\
& \left.+(-1)^{(m-1 / 2)} D_{x} P_{m n} K_{n}-\frac{\alpha_{m}}{2} D_{x} L_{n}\right],
\end{aligned}
$$

where $C_{m n}=\left(1 /\left(D_{x}\left(\alpha_{m}^{4} / 16\right)+H\left(\alpha_{m}^{2} \beta_{n}^{2} / 8\right)+D_{y}\left(\beta_{n}^{4} / 16\right)\right)\right)$, $R_{m n}=\mu_{x}\left(\alpha_{m}^{2} / 4\right)+\left(\beta_{n}^{2} / 4\right)$, and $P_{m n}=\left(\alpha_{m}^{2} / 4\right)+\mu_{y}\left(\beta_{n}^{2} / 4\right)$.

By substituting equation (18) into equation (6), the expression for $W(x, y)$ is obtained as follows for $m=1,3,5, \ldots \infty$ and $n=1,3,5, \ldots \infty$ :

$$
W(x, y)=\frac{4}{a b} \sum_{m=1,3}^{\infty} \sum_{n=1,3}^{\infty} C_{m n}\left[\begin{array}{c}
q_{m n}+(-1)^{(n-1 / 2)} D_{y} R_{m n} I_{m}-D_{y} \frac{\beta_{n}}{2} J_{m} \\
+(-1)^{(m-1 / 2)} D_{x} P_{m n} K_{n}-\frac{\alpha_{m}}{2} D_{x} L_{n}
\end{array}\right] \sin \frac{\alpha_{m} x}{2} \sin \frac{\beta_{n} y}{2} .
$$

Case 1. For the CCFF rectangular plate, equation (19) has satisfied the boundary conditions $\left.W\right|_{x=0}=\left.W\right|_{y=0}=0$, $\left.V_{x}\right|_{x=a}=\left.V_{y}\right|_{y=b}=0, \quad$ and $\quad \partial W^{2} /\left.\partial x \partial y\right|_{x=a, y=b}=0 . \quad$ By substituting $W(x, y)$ into the other boundary conditions $\partial W /\left.\partial x\right|_{x=0}=\partial W /\left.\partial y\right|_{y=0}=0$ and $\left.M_{x}\right|_{x=a}=\left.M_{y}\right|_{y=b}=0$, and taking the differentiation technique of trigonometric series [41], we can obtain 


$$
\begin{aligned}
& \sum_{m=1,3}^{\infty}(-1)^{(n-1 / 2)} D_{y} C_{m n} R_{m n} \alpha_{m} I_{m}-\sum_{m=1,3}^{\infty} D_{y} C_{m n} \alpha_{m} \frac{\beta_{n}}{2} J_{m}+\sum_{m=1,3}^{\infty}(-1)^{(m-1 / 2)} D_{x} C_{m n} P_{m n} \alpha_{m} K_{n} \\
& -\sum_{m=1,3}^{\infty} D_{x} C_{m n} \alpha_{m} \frac{\alpha_{m}}{2} L_{n}=-\sum_{m=1,3}^{\infty} C_{m n} \alpha_{m} q_{m n} \quad n=1,3,5 \ldots \\
& \sum_{m=1,3}^{\infty}(-1)^{(m-1 / 2)}(-1)^{(n-1 / 2)}\left[\mu_{y}-D_{y} C_{m n} R_{m n} P_{m n}\right] I_{m}+\sum_{m=1,3}^{\infty}(-1)^{(m-1 / 2)} D_{y} C_{m n} P_{m n} \frac{\beta_{n}}{2} J_{m} \\
& +\sum_{m=1,3}^{\infty}(-1)^{m-1}\left[1-D_{x} C_{m n} P_{m n}^{2}\right] K_{n} \\
& +\sum_{m=1,3}^{\infty}(-1)^{(m-1 / 2)} D_{x} C_{m n} P_{m n} \frac{\alpha_{m}}{2} L_{n}=\sum_{m=1,3}^{\infty}(-1)^{(m-1 / 2)} C_{m n} P_{m n} q_{m n} \quad n=1,3,5 \ldots, \\
& \sum_{n=1,3}^{\infty}(-1)^{(n-1 / 2)} D_{y} C_{m n} R_{m n} \beta_{n} I_{m}-\sum_{n=1,3}^{\infty} D_{y} C_{m n} \beta_{n} \frac{\beta_{n}}{2} J_{m}+\sum_{n=1,3}^{\infty}(-1)^{(m-1 / 2)} D_{x} C_{m n} P_{m n} \beta_{n} K_{n} \\
& -\sum_{n=1,3}^{\infty} D_{x} C_{m n} \beta_{n} \frac{\alpha_{m}}{2} L_{n}=-\sum_{n=1,3}^{\infty} C_{m n} \beta_{n} q_{m n} \quad m=1,3,5 \ldots \\
& \sum_{n=1,3}^{\infty}(-1)^{n-1}\left[1-D_{y} C_{m n} R_{m n}^{2}\right] I_{m}+\sum_{n=1,3}^{\infty}(-1)^{(n-1 / 2)} D_{y} C_{m n} R_{m n} \frac{\beta_{n}}{2} J_{m} \\
& +\sum_{n=1,3}^{\infty}(-1)^{(n-1 / 2)}(-1)^{(m-1 / 2)}\left[\mu_{x}-D_{x} C_{m n} R_{m n} P_{m n}\right] K_{n} \\
& +\sum_{n=1,3}^{\infty}(-1)^{(n-1 / 2)} D_{x} C_{m n} R_{m n} \frac{\alpha_{m}}{2} L_{n}=\sum_{n=1,3}^{\infty}(-1)^{(n-1 / 2)} C_{m n} R_{m n} q_{m n} \quad m=1,3,5 \ldots,
\end{aligned}
$$

The constants $I_{m}, J_{m}, K_{n}$, and $L_{n}(m, n=1,3,5, \ldots)$ can be obtained by solving the infinite linear simultaneous equations described by equations (20)-(23). In calculation, a finite number of terms are taken in each set of equations and solved for a finite number of constants, i.e., $m=1,3,5, \ldots$, $M$, and $n=1,3,5, \ldots, N$, where $M$ and $N$ are any positive integers. Here, the same term $t$ is chosen for $m$ and $n$, with their upper limit taken as $M=(2 t-1) / 2$ and $N=(2 t-1) / 2$. By substituting the above constant solutions into equation (19), we finally get the analytical bending solutions for plate with two adjacent edges free and the other two edges clamped.

The bending moment along the clamped edge can be easily found by the following equation:

$$
\begin{aligned}
& M_{x}=-\left.D_{x}\left(\frac{\partial^{2} W}{\partial x^{2}}+\mu_{y} \frac{\partial^{2} w}{\partial y^{2}}\right)\right|_{x=0}=-\left.D_{x} \frac{\partial^{2} W}{\partial x^{2}}\right|_{x=0}=-D_{x} \frac{2}{b} \sum_{n=1,3}^{\infty} L_{n} \sin \frac{\beta_{n} y}{2}, \\
& M_{y}=-\left.D_{y}\left(\frac{\partial^{2} W}{\partial y^{2}}+\mu_{x} \frac{\partial^{2} W}{\partial x^{2}}\right)\right|_{y=0}=-\left.D_{y} \frac{\partial^{2} W}{\partial y^{2}}\right|_{y=0}=-D_{y} \frac{2}{a} \sum_{n=1,3}^{\infty} J_{m} \sin \frac{\alpha_{m} x}{2} .
\end{aligned}
$$

Case 2. For the rectangular plate clamped at edge $x=0$, simply supported at edge $y=0$, and free at edges $x=a$ and $y=b$, the undetermined unknowns $J_{m}$ will be zero, and the deflection of the plate reduces to

$$
W(x, y)=\frac{4}{a b} \sum_{m=1,3}^{\infty} \sum_{n=1,3}^{\infty} C_{m n}\left[\begin{array}{c}
q_{m n}+(-1)^{(n-1 / 2)} D_{y} R_{m n} I_{m} \\
+(-1)^{(m-1 / 2)} D_{x} P_{m n} K_{n}-\frac{\alpha_{m}}{2} D_{x} L_{n}
\end{array}\right] \sin \frac{\alpha_{m} x}{2} \sin \frac{\beta_{n} y}{2} .
$$


By substituting $W(x, y)$ into the other boundary conditions $\partial W /\left.\partial x\right|_{x=0}=0$ and $\left.M_{x}\right|_{x=a}=\left.M_{y}\right|_{y=b}=0$, and taking the differentiation technique of trigonometric series [41], we achieved

$$
\begin{aligned}
& \sum_{m=1,3}^{\infty}(-1)^{(n-1 / 2)} D_{y} C_{m n} R_{m n} \alpha_{m} I_{m}+\sum_{m=1,3}^{\infty}(-1)^{(m-1 / 2)} D_{x} C_{m n} P_{m n} \alpha_{m} K_{n}-\sum_{m=1,3}^{\infty} D_{x} C_{m n} \alpha_{m} \frac{\alpha_{m}}{2} L_{n} \\
& =-\sum_{m=1,3}^{\infty} C_{m n} \alpha_{m} q_{m n} \quad n=1,3,5 \ldots, \\
& \sum_{m=1,3}^{\infty}(-1)^{(m-1 / 2)}(-1)^{(n-1 / 2)}\left[\mu_{y}-D_{y} C_{m n} R_{m n} P_{m n}\right] I_{m}+\sum_{m=1,3}^{\infty}(-1)^{m-1}\left[1-D_{x} C_{m n} P_{m n}^{2}\right] K_{n} \\
& \quad+\sum_{m=1,3}^{\infty}(-1)^{(m-1 / 2)} D_{x} C_{m n} P_{m n} \frac{\alpha_{m}}{2} L_{n}=\sum_{m=1,3}^{\infty}(-1)^{(m-1 / 2)} C_{m n} P_{m n} q_{m n} \quad n=1,3,5 \ldots, \\
& \sum_{n=1,3}^{\infty}(-1)^{n-1}\left[1-D_{y} C_{m n} R_{m n}^{2}\right] I_{m}+\sum_{n=1,3}^{\infty}(-1)^{(n-1 / 2)}(-1)^{(m-1 / 2)}\left[\mu_{x}-D_{x} C_{m n} R_{m n} P_{m n}\right] K_{n} \\
& \quad+\sum_{n=1,3}^{\infty}(-1)^{(n-1 / 2)} D_{x} C_{m n} R_{m n} \frac{\alpha_{m}}{2} L_{n}=\sum_{n=1,3}^{\infty}(-1)^{(n-1 / 2)} C_{m n} R_{m n} q_{m n} \quad m=1,3,5 \ldots
\end{aligned}
$$

The constants $I_{m}, K_{n}$, and $L_{n}(m, n=1,3,5, \ldots)$ can be obtained by solving the infinite linear simultaneous equations described by equations (26)-(28) in a similar way to that in the Case 1 .
Case 3. For the rectangular plate simply supported at edges $x=0$ and $y=0$ and free at edges $x=a$ and $y=b$, the undetermined unknowns $J_{m}$ and $L_{n}$ will be zero, and the deflection of the plate reduces to

$$
W(x, y)=\frac{4}{a b} \sum_{m=1,3}^{\infty} \sum_{n=1,3}^{\infty} C_{m n}\left[q_{m n}+(-1)^{(n-1 / 2)} D_{y} R_{m n} I_{m}+(-1)^{(m-1 / 2)} D_{x} P_{m n} K_{n}\right] \sin \frac{\alpha_{m} x}{2} \sin \frac{\beta_{n} y}{2} .
$$

Equation (29) has to satisfy the remaining boundary conditions $\left.M_{x}\right|_{x=a}=\left.M_{y}\right|_{y=b}=0$, and by taking the differentiation technique of trigonometric series [41], we can get

$$
\begin{aligned}
& \sum_{m=1,3}^{\infty}(-1)^{(m-1 / 2)}(-1)^{(n-1 / 2)}\left[\mu_{y}-D_{y} C_{m n} R_{m n} P_{m n}\right] I_{m}+\sum_{m=1,3}^{\infty}(-1)^{m-1}\left[1-D_{x} C_{m n} P_{m n}^{2}\right] K_{n} \\
& \quad=\sum_{m=1,3}^{\infty}(-1)^{(m-1 / 2)} C_{m n} P_{m n} q_{m n} \quad n=1,3,5 \ldots, \\
& \sum_{n=1,3}^{\infty}(-1)^{n-1}\left[1-D_{y} C_{m n} R_{m n}^{2}\right] I_{m}+\sum_{n=1,3}^{\infty}(-1)^{(n-1 / 2)}(-1)^{(m-1 / 2)}\left[\mu_{x}-D_{x} C_{m n} R_{m n} P_{m n}\right] K_{n} \\
& =\sum_{n=1,3}^{\infty}(-1)^{(n-1 / 2)} C_{m n} R_{m n} q_{m n} \quad m=1,3,5 \ldots
\end{aligned}
$$

The unknown constants $I_{m}$ and $K_{n}(m, n=1,3,5, \ldots)$ can be obtained by solving equations (30)-(31) in a similar way to that in the Case 1 section.

\section{Numerical Results and Discussion}

Comprehensive examinations on the isotropic/orthotropic rectangular plates under three different support conditions are 
TABLE 1: Deflections and bending moments of CCFF isotropic square plate subjected to uniform loading with various Poisson's ratios.

\begin{tabular}{|c|c|c|c|c|c|c|c|c|c|}
\hline \multirow{2}{*}{$\begin{array}{l}\text { Poisson's ratio } \\
\mu\end{array}$} & \multirow{2}{*}{ Method } & \multirow{2}{*}{$\begin{array}{c}\text { Number of } \\
\text { terms }\end{array}$} & \multicolumn{4}{|c|}{$W\left(q_{0} a^{4} / D\right), y=b$} & \multicolumn{3}{|c|}{$M_{y}\left(q_{0} a^{2}\right), y=0$} \\
\hline & & & $x=a$ & $x=0.75 a$ & $x=0.50 a$ & $x=0.25 a$ & $x=0.75 a$ & $x=0.50 a$ & $x=0.25 a$ \\
\hline \multirow{11}{*}{0} & & $t=10$ & 0.034972 & 0.027382 & 0.017034 & 0.0060965 & -0.20029 & -0.12256 & -0.041199 \\
\hline & & $t=30$ & 0.035778 & 0.027647 & 0.017274 & 0.0062238 & -0.19607 & -0.12255 & -0.042982 \\
\hline & & $t=50$ & 0.035943 & 0.027730 & 0.017321 & 0.0062381 & -0.19730 & -0.12254 & -0.042474 \\
\hline & Present & $t=100$ & 0.036067 & 0.027780 & 0.017356 & 0.0062543 & -0.19689 & -0.12254 & -0.042645 \\
\hline & & $t=200$ & 0.036129 & 0.027807 & 0.017374 & 0.0062618 & -0.19689 & -0.12254 & -0.042644 \\
\hline & & $t=300$ & 0.036150 & 0.027816 & 0.017380 & 0.0062643 & -0.19689 & -0.12254 & -0.042644 \\
\hline & & $t=500$ & 0.036166 & 0.027823 & 0.017384 & 0.0062660 & -0.19689 & -0.12254 & -0.042644 \\
\hline & Liu and Li [42] & & 0.036237 & - & - & - & -0.19284 & - & -0.042656 \\
\hline & $\begin{array}{l}\text { Huang and } \\
\text { Conway [43] }\end{array}$ & & 0.03619 & - & - & - & - & - & - \\
\hline & FEM & & 0.036196 & 0.027836 & 0.017392 & 0.0062687 & -0.19499 & -0.12166 & -0.042779 \\
\hline & & & $x=a$ & $x=0.75 a$ & $x=0.50 a$ & $x=0.25 a$ & - & - & - \\
\hline \multirow{11}{*}{0.3} & & $t=10$ & 0.041986 & 0.032229 & 0.019507 & 0.0066292 & -0.23929 & -0.16565 & -0.095009 \\
\hline & & $t=30$ & 0.043062 & 0.032583 & 0.019801 & 0.0067455 & -0.23910 & -0.16552 & -0.094859 \\
\hline & & $t=50$ & 0.043278 & 0.032691 & 0.019858 & 0.0067528 & -0.23898 & -0.16549 & -0.094845 \\
\hline & Present & $t=100$ & 0.043441 & 0.032756 & 0.019901 & 0.0067648 & -0.23892 & -0.16548 & -0.094837 \\
\hline & & $t=200$ & 0.043523 & 0.032791 & 0.019922 & 0.0067700 & -0.23889 & -0.16547 & -0.094835 \\
\hline & & $t=300$ & 0.043550 & 0.032803 & 0.019930 & 0.0067717 & -0.23889 & -0.16547 & -0.094834 \\
\hline & & $t=500$ & 0.043572 & 0.032812 & 0.019935 & 0.0067728 & -0.23889 & -0.16547 & -0.094834 \\
\hline & Liu and $\mathrm{Li}[42]$ & & 0.043601 & 0.032828 & 0.019945 & 0.006774 & -0.242318 & - & -0.096808 \\
\hline & Fo-van [44] & & 0.043678 & 0.032872 & 0.019993 & 0.006771 & -0.23400 & - & -0.09102 \\
\hline & FEM & & 0.043611 & 0.032829 & 0.019945 & 0.0067745 & -0.23650 & -0.16426 & -0.094810 \\
\hline & & & $x=a$ & $x=0.75 a$ & $x=0.50 a$ & $x=0.25 a$ & - & - & - \\
\hline \multirow{10}{*}{$1 / 3$} & & $t=10$ & 0.043044 & 0.032965 & 0.019878 & 0.0067015 & -0.21694 & -0.13156 & -0.045939 \\
\hline & & $t=30$ & 0.044156 & 0.033329 & 0.020178 & 0.0068145 & -0.22696 & -0.13164 & -0.041677 \\
\hline & & $t=50$ & 0.044379 & 0.033441 & 0.020236 & 0.0068203 & -0.21987 & -0.13165 & -0.044603 \\
\hline & Present & $t=100$ & 0.044548 & 0.033508 & 0.020280 & 0.0068314 & -0.22272 & -0.13167 & -0.043406 \\
\hline & & $t=200$ & 0.044632 & 0.033544 & 0.020302 & 0.0068362 & -0.22274 & -0.13166 & -0.043410 \\
\hline & & $t=300$ & 0.044660 & 0.033556 & 0.020309 & 0.0068378 & -0.22274 & -0.13166 & -0.043409 \\
\hline & & $t=500$ & 0.044668 & 0.033566 & 0.020315 & 0.0068391 & -0.22274 & -0.13166 & -0.043409 \\
\hline & Liu and $\mathrm{Li}[42]$ & & 0.044665 & 0.033579 & 0.020316 & 0.006787 & -0.225449 & - & -0.042035 \\
\hline & $\begin{array}{c}\text { Leissa and } \\
\text { Niedenfuhr [45] }\end{array}$ & & 0.04055 & - & 0.018969 & - & -0.20753 & - & -0.04332 \\
\hline & FEM & & 0.044709 & 0.033573 & 0.020319 & 0.0068382 & -0.22071 & -0.13103 & -0.042797 \\
\hline
\end{tabular}

conducted to validate the present approach and examine the accuracy of the obtained results. The selected aspect ratio $b / a$ for rectangular plates ranges from 0.5 to 4 . Parametric analysis (effects of aspect ratios, Poisson's ratio, and boundary restraint) on bending characteristics of CCFF, CSFF, and SSFF isotropic/ orthotropic rectangular plates is also conducted. It is noteworthy that, for an isotropic plate, $H=D_{x}=D_{y}=D$, $D_{x y}=(1-\mu) D / 2$, and $\mu_{x}=\mu_{y}=\mu$ where $\mu$ and $D$ are Poisson's ratio and flexural stiffness of plate, respectively.

(1) An isotropic square CCFF plate with various Poison ratios, under uniform load $\left(q(x, y)=q_{0}\right)$

(2) An orthotropic rectangular CCFF plate under linearly varying load $\left(q(x, y)=q_{0} \cdot x / a\right)$, where $D_{y}=4 D_{x}, D_{x y}=0.85 D_{x}, \mu_{x}=0.075$, and $\mu_{y}=0.3$.

(3) An isotropic rectangular CSFF plate with Poison ratio $\mu=1 / 3$, under uniform load $\left(q(x, y)=q_{0}\right)$.

(4) An orthotropic rectangular CSFF plate under central concentrate load $(q(x, y)=P)$, where $D_{y}=4 D_{x}$, $D_{x y}=0.85 D_{x}, \mu_{x}=0.075$, and $\mu_{y}=0.3$.
(5) An isotropic rectangular SSFF plate with Poison ratio $\mu=1 / 3$, subjected to a uniform load $\left(q(x, y)=q_{0}\right)$.

(6) An orthotropic rectangular SSFF plate under sinusoidal load $\left(q(x, y)=q_{0} \sin (\pi x / a)\right)$, where $D_{y}=4 D_{x}, D_{x y}=0.85 D_{x}, \mu_{x}=0.075$, and $\mu_{y}=0.3$.

As shown in Table 1, the obtained nondimensional bending solutions (deflections and bending moments at specific points) of CCFF square isotropic rectangular plates increased with the increase of Poisson's ratio. It is clear that the obtained bending solutions of SSFF plate are always larger than those of CSFF and CCFF plates for each aspect ratio; the bending solutions of CSFF plate is always larger than those of CCFF plate, which indicates plates with clamped edges possess lower deform capability than plates with simply supported edges. Finally, it is also observed that the bending solutions of isotropic/orthotropic plates under three different support conditions decreased with the decreasing aspect ratio. The above parametric studies reveal that aspect ratios, Poisson's ratio, and boundary restraint have a significant influence on the bending characteristics of plates. 
TABLe 2: Deflections and bending moments of CCFF orthotropic rectangular plate under linear loading $\left(D_{y}=4 D_{x}, D_{x y}=0.85 D_{x}\right.$, $\left.\mu_{x}=0.075, \mu_{y}=0.3\right)$.

\begin{tabular}{|c|c|c|c|c|c|c|c|c|}
\hline \multirow{2}{*}{ Method } & \multirow{2}{*}{$b / a$} & \multicolumn{4}{|c|}{$W\left(q_{0} a^{4} / D_{x}\right), y=b$} & \multicolumn{3}{|c|}{$M_{x}\left(q_{0} a^{2}\right), x=0$} \\
\hline & & $x=a$ & $x=0.75 a$ & $x=0.50 a$ & $x=0.25 a$ & $y=0.75 b$ & $y=0.50 b$ & $y=0.25 b$ \\
\hline Present & \multirow{2}{*}{0.5} & 0.0014045 & 0.0011974 & 0.00081803 & 0.00033817 & -0.012265 & -0.0052803 & -0.00077096 \\
\hline FEM & & 0.0014051 & 0.0011978 & 0.00081832 & 0.00033825 & -0.012287 & -0.0052763 & -0.00079197 \\
\hline Present & \multirow{2}{*}{1.0} & 0.012793 & 0.010023 & 0.0062899 & 0.0022584 & -0.065799 & -0.032091 & -0.0064661 \\
\hline FEM & & 0.012804 & 0.010028 & 0.0062931 & 0.0022593 & -0.065586 & -0.031816 & -0.0064944 \\
\hline Present & \multirow{2}{*}{2.0} & 0.051082 & 0.035948 & 0.020085 & 0.0062465 & -0.019256 & -0.12485 & -0.039702 \\
\hline FEM & & 0.051098 & 0.035953 & 0.020082 & 0.0062464 & -0.19107 & -0.12423 & -0.039649 \\
\hline Present & \multirow{2}{*}{3.0} & 0.075008 & 0.050613 & 0.026856 & 0.0078044 & -0.27312 & -0.21195 & -0.089978 \\
\hline FEM & & 0.075010 & 0.050615 & 0.026855 & 0.0078025 & -0.27087 & -0.21034 & -0.090173 \\
\hline Present & \multirow{2}{*}{4.0} & 0.084869 & 0.056324 & 0.029256 & 0.0082630 & -0.30949 & -0.26530 & -0.13949 \\
\hline FEM & & 0.084838 & 0.056321 & 0.029253 & 0.0082595 & -0.30702 & -0.26318 & -0.13898 \\
\hline
\end{tabular}

TABLE 3: Deflections and bending moments of CSFF isotropic square plate under uniform loading with Poisson's ratio $\mu=1 / 3$.

\begin{tabular}{|c|c|c|c|c|c|c|c|c|}
\hline \multirow{2}{*}{ Method } & \multirow{2}{*}{$b / a$} & \multicolumn{4}{|c|}{$W\left(q_{0} a^{4} / D_{x}\right), y=b$} & \multicolumn{3}{|c|}{$M_{x}\left(q_{0} a^{2}\right), x=0$} \\
\hline & & $x=a$ & $x=0.75 a$ & $x=0.50 a$ & $x=0.25 a$ & $y=0.75 b$ & $y=0.50 b$ & $y=0.25 b$ \\
\hline Present & \multirow{2}{*}{0.5} & 0.029137 & 0.023545 & 0.015447 & 0.0057435 & -0.21714 & -0.14227 & -0.074437 \\
\hline FEM & & 0.029145 & 0.023548 & 0.015448 & 0.0057428 & -0.21621 & -0.14211 & -0.074837 \\
\hline Present & \multirow{2}{*}{1.0} & 0.073524 & 0.052971 & 0.030484 & 0.0096223 & -0.34599 & -0.25601 & -0.14954 \\
\hline FEM & & 0.073563 & 0.052976 & 0.030485 & 0.0096193 & -0.34440 & -0.25567 & -0.14891 \\
\hline Present & \multirow{2}{*}{2.0} & 0.11539 & 0.077393 & 0.040624 & 0.011296 & -0.47278 & -0.41166 & -0.27960 \\
\hline FEM & & 0.11540 & 0.077372 & 0.040600 & 0.011286 & -0.46779 & -0.40738 & -0.27711 \\
\hline Present & \multirow{2}{*}{3.0} & 0.12262 & 0.081040 & 0.041686 & 0.011250 & -0.50257 & -0.46955 & -0.35885 \\
\hline FEM & & 0.12261 & 0.081010 & 0.041663 & 0.011234 & -0.49749 & -0.46475 & -0.35525 \\
\hline Present & \multirow{2}{*}{4.0} & 0.12347 & 0.081403 & 0.041722 & 0.011201 & -0.50624 & -0.48994 & -0.41077 \\
\hline FEM & & 0.12347 & 0.081369 & 0.041695 & 0.011182 & -0.50116 & -0.48499 & -0.40652 \\
\hline
\end{tabular}

TABle 4: Deflections and bending moments of CSFF orthotropic square plate subjected to central concentrated load $\left(D_{y}=4 D_{x}, D_{x y}=0.85 D_{x}, \mu_{x}=0.075, \mu_{y}=0.3\right)$.

\begin{tabular}{lcccccccc}
\hline \multirow{2}{*}{ Method } & \multirow{2}{*}{$b / a$} & $x=a$ & $x=0.75 a$ & $x=0.50 a$ & $x=0.25 a$ & $y=0.75 b$ & $y=0.50 b$ & $y=0.25 b$ \\
\hline Present & \multirow{2}{*}{0.5} & 0.025644 & 0.024551 & 0.019873 & 0.0084041 & -0.33149 & -0.21000 & -0.10442 \\
FEM & & 0.025655 & 0.024562 & 0.019880 & 0.0084065 & -0.33043 & -0.21026 & -0.10339 \\
Present & \multirow{2}{*}{1.0} & 0.035436 & 0.029660 & 0.020200 & 0.0073935 & -0.28753 & -0.22909 & -0.12999 \\
FEM & & 0.035458 & 0.029670 & 0.020204 & 0.0073936 & -0.28347 & -0.22620 & -0.12963 \\
Present & \multirow{2}{*}{2.0} & 0.033743 & 0.023512 & 0.012874 & 0.0037013 & -0.20785 & -0.25596 & -0.14027 \\
FEM & & 0.033746 & 0.023510 & 0.012864 & 0.0036989 & -0.20600 & -0.25154 & -0.13967 \\
Present & \multirow{2}{*}{3.0} & 0.023279 & 0.014747 & 0.0070937 & 0.0016934 & -0.14649 & -0.26115 & -0.11393 \\
FEM & & 0.023271 & 0.014741 & 0.0070888 & 0.0016910 & -0.14595 & -0.25654 & -0.11378 \\
Present & \multirow{2}{*}{4.0} & 0.014617 & 0.0087457 & 0.0038585 & 0.00079266 & -0.10166 & -0.25972 & -0.084991 \\
FEM & & 0.014602 & 0.0087393 & 0.0038539 & 0.00079059 & -0.10103 & -0.25506 & -0.085080 \\
\hline
\end{tabular}

Through Tables $1-6$, it can be seen that the obtained results of deflections and bending moments agree very well with those from the literature and, especially, those by FEM results. The validity of the employed method and the accuracy of the obtained results are illuminated through the above examples, which means that the present approach is competent for analyzing the bending problems of orthotropic rectangular thin plates with the title boundary condition.

Table 1 shows the convergence study and results for deflection and bending moment obtained with the accuracy of five significant figures. It is theoretically exact for the results when $t \longrightarrow \infty$, while in the present paper the obtained analytical results suffice for the title problem by taking the series terms $(t=500)$. It is beyond the expectation that convergence rates of the bending moments are faster than ones of the transverse deflection. This is attributing to the fact that the present deflection results are at free edges. The slower convergent results are due to the final solution expressed by double sine Fourier series. However, the linear simultaneous equations can be easily calculated via Mathematica software, and above all, the value of the employed method lies in its merits of rational solution procedure and simple mathematical manipulation that 
TABLE 5: Deflections and bending moments of SSFF isotropic square plate with Poisson's ratio subjected to uniform loading.

\begin{tabular}{|c|c|c|c|c|c|c|c|c|}
\hline \multirow{2}{*}{ Method } & \multirow{2}{*}{$b / a$} & \multicolumn{4}{|c|}{$W\left(q_{0} a^{4} / D\right), y=b$} & \multicolumn{3}{|c|}{$W\left(q_{0} a^{4} / D\right)$} \\
\hline & & $x=a$ & $x=0.75 a$ & $x=0.50 a$ & $x=0.25 a$ & $(0.75 a, 0.75 b)$ & $(0.50 a, 0.50 b)$ & $(0.25 a, 0.25 b)$ \\
\hline Present & \multirow{3}{*}{0.5} & 0.046862 & 0.040536 & 0.030864 & 0.017032 & 0.030728 & 0.015757 & 0.0044281 \\
\hline FEM & & 0.046879 & 0.040547 & 0.030872 & 0.017038 & 0.030740 & 0.015758 & 0.0044300 \\
\hline Present & & 0.18750 & 0.150924 & 0.107884 & 0.056845 & 0.119112 & 0.059318 & 0.016342 \\
\hline Liu, 2010 & \multirow{3}{*}{1.0} & 0.18750 & 0.150894 & 0.107868 & 0.056839 & 0.119112 & 0.059318 & 0.016342 \\
\hline Leissa, 1963 & & 0.19276 & - & 0.110819 & - & 0.122306 & 0.060857 & 0.016746 \\
\hline FEM & & 0.18749 & 0.150885 & 0.107861 & 0.056836 & 0.119103 & 0.059318 & 0.016340 \\
\hline Present & \multirow{2}{*}{2.0} & 0.74979 & 0.57559 & 0.39293 & 0.20013 & 0.49165 & 0.25212 & 0.070849 \\
\hline FEM & & 0.74984 & 0.57559 & 0.39294 & 0.20013 & 0.49159 & 0.25206 & 0.070841 \\
\hline Present & \multirow{4}{*}{3.0} & 1.6871 & 1.2791 & 0.86226 & 0.43487 & 1.1372 & 0.59568 & 0.16980 \\
\hline FEM & & 1.6871 & 1.2790 & 0.86222 & 0.43486 & 1.1370 & 0.59560 & 0.16978 \\
\hline Present & & 2.9993 & 2.2635 & 1.5186 & 0.76303 & 2.0525 & 1.0850 & 0.31153 \\
\hline FEM & & 2.9992 & 2.2633 & 1.5184 & 0.76298 & 2.0522 & 1.0848 & 0.31150 \\
\hline
\end{tabular}

TABLE 6: Deflections of SSFF orthotropic square plate subjected to sinusoidal load $\left(D_{y}=4 D_{x}, D_{x y}=0.85 D_{x}, \mu_{x}=0.075, \mu_{y}=0.3\right)$.

\begin{tabular}{lcccccccc}
\hline \multirow{2}{*}{ Method } & \multirow{2}{*}{$\boldsymbol{b} / a$} & $x=a$ & $x=0.75 a$ & $x=0.50 a$ & $x=0.25 a$ & $(0.75 a, 0.75 b)$ & $\begin{array}{c}W\left(q_{0} a^{4} / D_{x}\right) \\
(0.50 a, 0.50 b)\end{array}$ \\
& & 0.011700 & 0.010881 & 0.0088150 & 0.0050191 & 0.0082315 & 0.0045234 & 0.0013139 \\
Present & \multirow{2}{*}{0.5} & 0.011708 & 0.010888 & 0.0088204 & 0.0050225 & 0.0082376 & 0.0045251 & 0.0013150 \\
FEM & & 0.046785 & 0.040375 & 0.030744 & 0.016855 & 0.031381 & 0.016617 & 0.0047686 \\
Present & \multirow{2}{*}{1.0} & 0.046825 & 0.040401 & 0.030764 & 0.016866 & 0.031389 & 0.016621 & 0.0047697 \\
FEM & & 0.18719 & 0.14885 & 0.10524 & 0.054918 & 0.12382 & 0.064574 & 0.018470 \\
Present & \multirow{2}{*}{2.0} & 0.18729 & 0.14890 & 0.10528 & 0.054937 & 0.12384 & 0.064584 & 0.018474 \\
FEM & & 0.42118 & 0.32521 & 0.22339 & 0.11420 & 0.28311 & 0.14924 & 0.043046 \\
Present & \multirow{2}{*}{3.0} & 0.42139 & 0.32531 & 0.22346 & 0.11424 & 0.28317 & 0.14927 & 0.043054 \\
FEM & & 0.74878 & 0.57114 & 0.38747 & 0.19629 & 0.51004 & 0.27077 & 0.078462 \\
Present & \multirow{2}{*}{4.0} & 0.74912 & 0.57130 & 0.38758 & 0.19635 & 0.51014 & 0.27083 & 0.078478 \\
FEM & & & & & & &
\end{tabular}

enable the method to serve as an easy-to-implement theoretical tool in exploring analytical bending solutions of a plate.

\section{Conclusion}

This paper investigates the analytical bending solution of orthotropic rectangular thin plates with two adjacent edges free and the others clamped or simply supported. The significant merits of the adopted method differing from typical semi-inverse ones are as follows: (1) it provides a rigorous and theoretical solution procedure for precise plate bending analysis without assuming the solution forms; (2) based on integral transform theory, it reduces the mathematical difficulty of plate problem by converting the boundary value problems of higher-order partial differential equation into solving linear algebra equations; (3) the present approach allows more precise analytical solutions for static problems of moderately thick and thick plates under similar boundary conditions. The obtained results are expected to serve as an accurate solution for validation of other numerical methods.

\section{Data Availability}

The data used to support the findings of this study are included within the article.

\section{Conflicts of Interest}

The authors declare that they have no conflicts of interest.

\section{Acknowledgments}

The work reported in this paper was supported by the Youth Fund of Colleges and Universities in Hebei Province Science and Technology Research Project (Grant no. QN2019024).

\section{References}

[1] M. Karimi and A. R. Shahidi, "Thermo-mechanical vibration, buckling, and bending of orthotropic graphene sheets based on nonlocal two-variable refined plate theory using finite difference method considering surface energy effects," Proceedings of the Institution of Mechanical Engineers, Part N: Journal of Nanomaterials, Nanoengineering and Nanosystems, vol. 231, no. 3, pp. 111-130, 2017.

[2] P. Shen and P. He, "Bending analysis of rectangular moderately thick plates using spline finite element method," Computers \& Structures, vol. 54, no. 6, pp. 1023-1029, 1995.

[3] J. B. Paiva, "Corner restrictions and their application to bending plate analyses by the boundary element method," Engineering Analysis with Boundary Elements, vol. 95, pp. 111, 2018.

[4] L. Sator, V. Sladek, and J. Sladek, "Bending of FGM plates under thermal load: classical thermoelasticity analysis by a 
meshless method," Composites Part B: Engineering, vol. 146, pp. 176-188, 2018.

[5] T. Li and R. K. Kapania, "On the formulation of a high-order discontinuous finite element method based on orthogonal polynomials for laminated plate structures," International Journal of Mechanical Sciences, vol. 149, pp. 530-548, 2018.

[6] Z. Fu, W. Chen, and W. Yang, "Winkler plate bending problems by a truly boundary-only boundary particle method," Computational Mechanics, vol. 44, no. 6, pp. 757763, 2009.

[7] G. S. Pavan and K. S. Nanjunda Rao, "Bending analysis of laminated composite plates using isogeometric collocation method," Composite Structures, vol. 176, pp. 715-728, 2017.

[8] Ö. Civalek, "Three-dimensional vibration, buckling and bending analyses of thick rectangular plates based on discrete singular convolution method," International Journal of $\mathrm{Me}$ chanical Sciences, vol. 49, no. 6, pp. 752-765, 2007.

[9] Ö. Civalek and M. H. Acar, "Discrete singular convolution method for the analysis of Mindlin plates on elastic foundations," International Journal of Pressure Vessels and Piping, vol. 84, no. 9, pp. 527-535, 2007.

[10] O. Civalek and E. Emsen, "Discrete singular convolution method for bending analysis of Reissner/Mindlin plates using geometric transformation," Steel \& Composite Structures, vol. 9, no. 1, pp. 59-75, 2009.

[11] Ö. Civalek and H. Ersoy, "Free vibration and bending analysis of circular Mindlin plates using singular convolution method," Communications in Numerical Methods in Engineering, vol. 25, no. 8, pp. 907-922, 2009.

[12] Ö. Civalek, "Application of differential quadrature (DQ) and harmonic differential quadrature (HDQ) for buckling analysis of thin isotropic plates and elastic columns," Engineering Structures, vol. 26, no. 2, pp. 171-186, 2004.

[13] O. Civalek and M. Ulker, "Harmonic differential quadrature (HDQ) for axisymmetric bending analysis of thin isotropic circular plates," Structural Engineering and Mechanics, vol. 17, no. 1, pp. 1-14, 2004.

[14] H.-T. Thai and D.-H. Choi, "Levy solution for free vibration analysis of functionally graded plates based on a refined plate theory," KSCE Journal of Civil Engineering, vol. 18, no. 6, pp. 1813-1824, 2014.

[15] H.-T. Thai and S.-E. Kim, "Analytical solution of a two variable refined plate theory for bending analysis of orthotropic Levy-type plates," International Journal of Mechanical Sciences, vol. 54, no. 1, pp. 269-276, 2012.

[16] H.-T. Thai and S.-E. Kim, "Levy-type solution for buckling analysis of orthotropic plates based on two variable refined plate theory," Composite Structures, vol. 93, no. 7, pp. 17381746, 2011.

[17] H.-T. Thai and S.-E. Kim, "Levy-type solution for free vibration analysis of orthotropic plates based on two variable refined plate theory," Applied Mathematical Modelling, vol. 36, no. 8, pp. 3870-3882, 2012.

[18] S. P. Timoshenko and J. M. Gere, Theory of Elastic Stability. 1961, McGrawHill-Kogakusha Ltd, Tokyo, Japan, 1961.

[19] K. K. Żur, "Green's function approach to frequency analysis of thin circular plates," Bulletin of the Polish Academy of Sciences Technical Sciences, vol. 64, no. 1, pp. 181-188, 2016.

[20] K. K. Zur, "Green's function for frequency analysis of thin annular plates with nonlinear variable thickness," Applied Mathematical Modelling, vol. 40, no. 5-6, pp. 3601-3619, 2016.

[21] K. K. Żur, "Green's function in frequency analysis of circular thin plates of variable thickness," Journal of Theoretical and Applied Mechanics, vol. 53, no. 4, pp. 873-884, 2015.
[22] C. W. LIM, "Symplectic elasticity approach for free vibration of rectangular plates," in International Conference on Vibration Engineering \& Technology of Machinery (VETOMAC IV), Hyderabad, India, December 2007.

[23] C. W. Lim, C. F. Lü, Y. Xiang, and W. Yao, "On new symplectic elasticity approach for exact free vibration solutions of rectangular Kirchhoff plates," International Journal of Engineering Science, vol. 47, no. 1, pp. 131-140, 2009.

[24] C. W. Lim and X. S. Xu, "Symplectic elasticity: theory and applications," Applied Mechanics Reviews, vol. 63, no. 5, Article ID 050802, 2010.

[25] C. W. Lim, W. A. Yao, and S. Cui, "Benchmark symplectic solutions for bending of corner-supported rectangular thin plates," The IES Journal Part A: Civil \& Structural Engineering, vol. 1, no. 2, pp. 106-115, 2008.

[26] R. Li, B. Wang, and P. Li, "Hamiltonian system-based benchmark bending solutions of rectangular thin plates with a corner point-supported," International Journal of Mechanical Sciences, vol. 85, pp. 212-218, 2014.

[27] R. Li, P. Wang, Y. Tian, B. Wang, and G. Li, "A unified analytic solution approach to static bending and free vibration problems of rectangular thin plates," Scientific Reports, vol. 5, no. $1,2015$.

[28] R. Li, X. Zheng, H. Wang, S. Xiong, K. Yan, and P. Li, "New analytic buckling solutions of rectangular thin plates with all edges free," International Journal of Mechanical Sciences, vol. 144, pp. 67-73, 2018.

[29] B. Wang, P. Li, and R. Li, "Symplectic superposition method for new analytic buckling solutions of rectangular thin plates," International Journal of Mechanical Sciences, vol. 119, pp. 432-441, 2016.

[30] R. Li, Y. Tian, P. Wang, Y. Shi, and B. Wang, "New analytic free vibration solutions of rectangular thin plates resting on multiple point supports," International Journal of Mechanical Sciences, vol. 110, pp. 53-61, 2016.

[31] R. Li, P. Wang, R. Xue, and X. Guo, "New analytic solutions for free vibration of rectangular thick plates with an edge free," International Journal of Mechanical Sciences, vol. 131132, pp. 179-190, 2017.

[32] X. Zheng, Y. Sun, M. Huang et al., "Symplectic superposition method-based new analytic bending solutions of cylindrical shell panels," International Journal of Mechanical Sciences, vol. 152, pp. 432-442, 2019.

[33] I. N. Sneddon, Application of Integral Transforms in the Theory of Elasticity, Springer-Verlag, New York, NY, USA, 1975.

[34] A. M. M. Bidgoli, A. R. Daneshmehr, and R. Kolahchi, "Analytical bending solution of fully clamped orthotropic rectangular plates resting on elastic foundations by the finite integral transform method," Journal of Applied and Computational Mechanics, vol. 1, no. 2, pp. 52-58, 2015.

[35] R. Li, Y. Zhong, B. Tian, and Y. Liu, "On the finite integral transform method for exact bending solutions of fully clamped orthotropic rectangular thin plates," Applied Mathematics Letters, vol. 22, no. 12, pp. 1821-1827, 2009.

[36] B. Tian, R. Li, and Y. Zhong, "Integral transform solutions to the bending problems of moderately thick rectangular plates with all edges free resting on elastic foundations," Applied Mathematical Modelling, vol. 39, no. 1, pp. 128-136, 2015.

[37] B. Tian, Y. Zhong, and R. Li, "Analytic bending solutions of rectangular cantilever thin plates," Archives of Civil and Mechanical Engineering, vol. 11, no. 4, pp. 1043-1052, 2011.

[38] Y. Zhong and J.-H. Yin, "Free vibration analysis of a plate on foundation with completely free boundary by finite integral 
transform method," Mechanics Research Communications, vol. 35, no. 4, pp. 268-275, 2008.

[39] Y. Zhong and Y. S. Zhang, "Free vibration of rectangular thin plate on elastic foundation with four edges free," Journal of Vibration Engineering, vol. 19, no. 4, pp. 566-570, 2006.

[40] Y. Zhong, X.-F. Zhao, and R. Li, "Free vibration analysis of rectangular cantilever plates by finite integral transform method," International Journal for Computational Methods in Engineering Science and Mechanics, vol. 14, no. 3, pp. 221-226, 2013.

[41] R. Li, B. Tian, and Y. Zhong, "Analytical bending solutions of free orthotropic rectangular thin plates under arbitrary loading," Meccanica, vol. 48, no. 10, pp. 2497-2510, 2013.

[42] Y. Liu and R. Li, "Accurate bending analysis of rectangular plates with two adjacent edges free and the others clamped or simply supported based on new symplectic approach," Applied Mathematical Modelling, vol. 34, no. 4, pp. 856-865, 2010.

[43] M. K. Huang and H. D. Conway, "Bending of a uniformly loaded rectangular plate with two adjacent edges clamped and the others either simply supported or free," Journal of Applied Mechanics-Transactions of the ASME, vol. 19, no. 4, pp. 451460, 1952.

[44] C. Fo-van, "Rectangular plates with two adjacent edges clamped and other two adjacent edges free," Acta Mechanica Solida Sinica, vol. 4, pp. 491-502, 1981.

[45] A. W. Leissa and F. W. Niedenfuhr, "Bending of a square plate with two adjacent edges free and the others clamped or simply supported," AIAA Journal, vol. 1, no. 1, pp. 116-120, 1963. 